\title{
Stereotactic navigation for the prepsoas oblique lateral lumbar interbody fusion: technical note and case series
}

\author{
Anthony M. DiGiorgio, DO, MHA, ${ }^{1,2}$ Caleb S. Edwards, BA, ${ }^{3}$ Michael S. Virk, MD, PhD, ${ }^{2}$ \\ Praveen V. Mummaneni, MD, ${ }^{2}$ and Dean Chou, MD²
}

${ }^{1}$ Department of Neurosurgery, Louisiana State University Health Sciences Center, New Orleans, Louisiana; and 2Department of Neurosurgery and ${ }^{3}$ School of Medicine, University of California, San Francisco, California

The prepsoas retroperitoneal approach is a minimally invasive technique used for anterior lumbar interbody fusion. The approach may have a more favorable risk profile than the transpsoas approach, decreasing the risks that come with dissecting through the psoas muscle. However, the oblique angle of the spine in the prepsoas approach can be disorienting and challenging. This technical report provides an overview of the use of navigation in prepsoas oblique lateral lumbar interbody fusion in a series of 49 patients.

https://thejns.org/doi/abs/10.3171/2017.5.FOCUS17168

KEY WORDS prepsoas lumbar interbody fusion; OLIF; lumbar fusion; minimally invasive spinal fusion; spinal navigation

$\mathrm{T}$ HE oblique, or prepsoas, retroperitoneal approach is one of the recent innovations in achieving anterior spinal access. ${ }^{4,7,12,15}$ This approach was first described by Mayer in 1997.6 Some surgeons favor this prepsoas approach because it offers the advantages of the transpsoas approach while avoiding the risks associated with traversing through the psoas muscle itself. The transpsoas approach can have complications such as femoral, genitofemoral, iliohypogastric, or ilioinguinal nerve injury and psoas hematoma. These complications can lead to sensory or motor changes to the thigh and leg. ${ }^{9-11,14}$

The oblique angle to the spine with this approach can be disorienting and a challenge to surgeons, especially since the transpsoas approach is based on true anteroposterior and lateral fluoroscopic views. Navigation may provide advantages that fluoroscopy may not, given the oblique nature of the approach. We present here our technique of using navigation and neuromonitoring for this procedure. We also performed a retrospective chart review to document outcomes and complications for patients who had this procedure done at the University of California, San Francisco.

\section{Clinical Methods}

\section{Chart Review}

Charts of patients who had undergone this technique were retrospectively reviewed. Patient-reported visual analog scale (VAS) scores for back and leg pain were record- ed from both the preoperative assessment and the latest postoperative notes. Complications, readmissions, length of stay, estimated blood loss, and surgical time were also recorded. Static radiographs were used for fusion assessment.

\section{Preoperative Workup}

Preoperative evaluation should include MRI and 36in standing radiographs; CT is an option if specific bony anatomy needs to be evaluated before surgery (Figs. 1 and 2). The location of the aorta, vena cava, iliac arteries, and iliac veins can also be evaluated to assess the corridor between the vessels and the spine; this corridor exists in most patients. ${ }^{8}$ It is critical to obtain 36 -in standing radiographs to preoperatively evaluate not only spinal alignment, but also the position of the iliac crest on the left side to ensure that the procedure can be done (Fig. 3). If the crest is too high and an L4-5 procedure is contemplated, the 36-in standing radiographs will inform the surgeon if the approach is possible. If lordosis needs to be restored or any coronal correction needs to be done, the procedure for either can be planned for preoperatively with the standing 36-in radiographs. Flexion-extension films are also obtained to identify abnormal movement.

\section{Operative Technique}

The patient is intubated, and neuromonitoring leads

ABBREVIATIONS EMG = electromyography; OR = operating room; PSIS = posterior superior iliac spine; VAS = visual analog scale.

SUBMITTED April 1, 2017. ACCEPTED May 23, 2017.

INCLUDE WHEN CITING DOI: 10.3171/2017.5.FOCUS17168. 


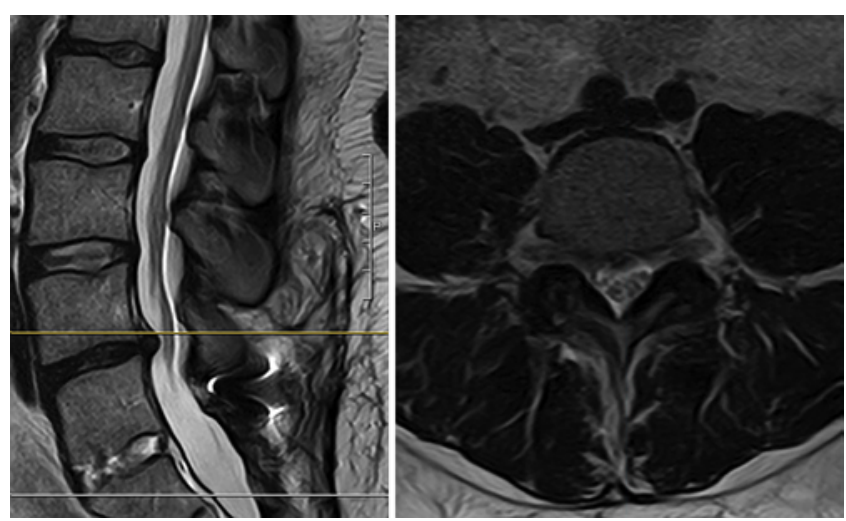

FIG. 1. Preoperative MR images obtained in a male patient with degenerative disease and foraminal stenosis at $L 4-5$. He had previously undergone an L5-S1 transforaminal lumbar interbody fusion at an outside institution.

are placed. We use the standard neuromonitoring that is available in the hospital, and we use motor evoked potentials as well as free-running electromyography (EMG). The patient is carefully placed in the right lateral decubitus position with the left side up (Fig. 4). Care must be taken to pad the common peroneal nerve and bony prominences. An axillary roll is used along the chest wall as well. The right side cannot be used with the prepsoas technique because of the vena cava obstructing the approach angle. If a right-sided approach must be performed, the traditional transpsoas approach should be performed since the prepsoas approach would present too high a risk of catastrophic vascular injury on the right side and should not be undertaken. The patient's arms need to be sufficiently out of the way to allow for intraoperative CT scanning.

The choice of anesthetic is critical for intraoperative neuromonitoring. We employ triggered EMG monitoring for the approach even though the theoretical risk of injury to the femoral nerve is low. Thus, paralytic medications cannot be used because they can lead to intraoperative false-negative triggered EMG recordings.

After positioning and draping the patient, the posterior superior iliac spine (PSIS) is identified. Moving 2 inches
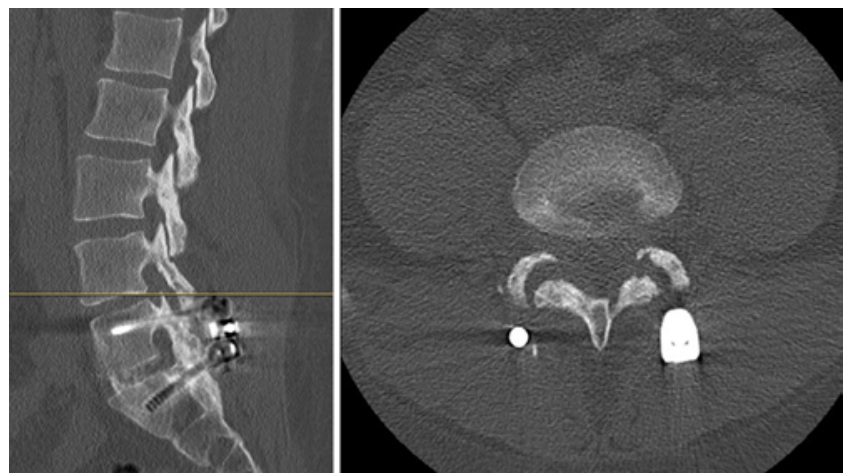

FIG. 2. Preoperative CT scans showing a solid posterolateral fusion at $\mathrm{L} 5-\mathrm{S} 1$ and widened facets at $\mathrm{L} 4-5$.
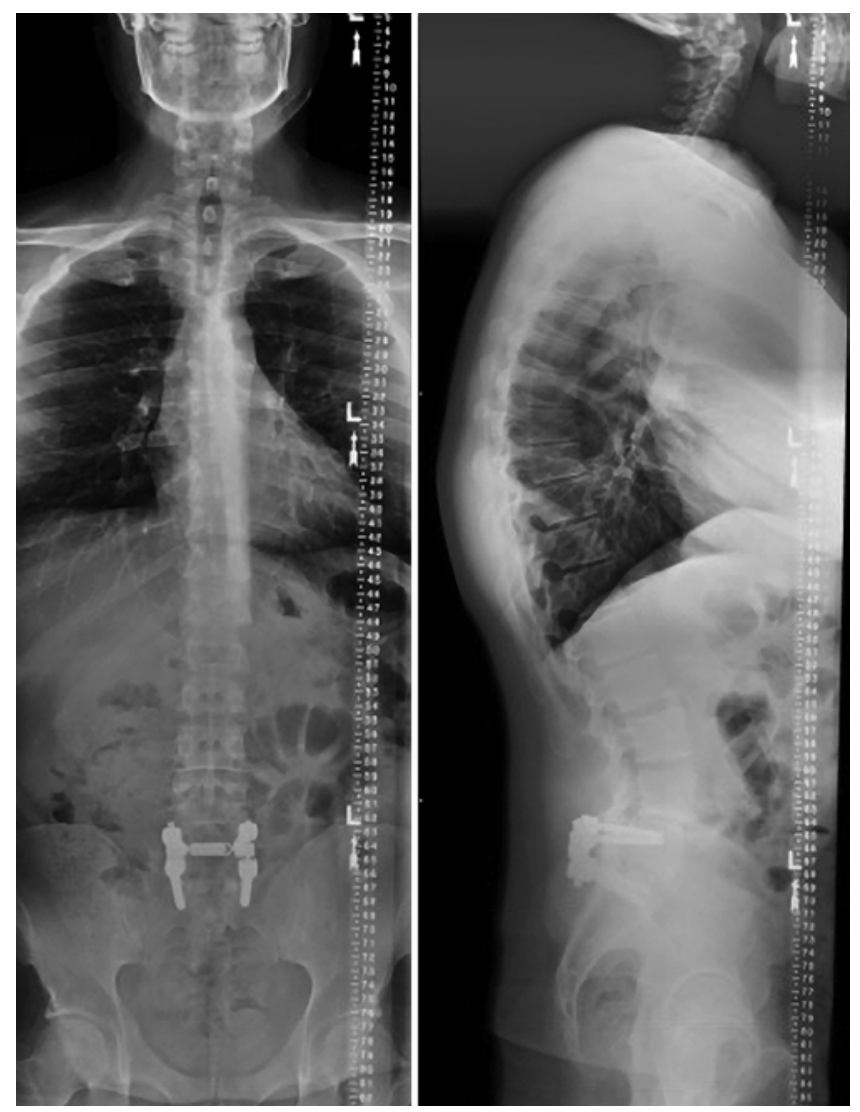

FIG. 3. Preoperative standing 36-in radiographs show the previously placed instrumentation at L5-S1 and spondylolisthesis at L4-5. The patient's global spinal alignment is normal.

superolaterally along the iliac crest, a $2-\mathrm{cm}$ incision is made, and the reference array for navigation is placed (Fig. 4 inset). This reference array needs to be securely placed in bone, out of the way of the surgeon so it is not inadvertently moved during the case.

Intraoperative CT images are obtained and registered to the navigation software (Fig. 5). This allows the incision to be planned using navigational assistance. Using a probe with virtual extension on navigation, we localize the midportion of the pathological disc space from a true lateral trajectory on the skin (Fig. 6). This is marked and a measurement is taken $5 \mathrm{~cm}$ anterior to this point. The incision is made in an oblique fashion, parallel to the abdominal wall nerve root trajectories.

The incision is carried down to the abdominal fascia with a trajectory toward the lumbar spine. Care must be taken not to enter the peritoneal cavity. The trajectory can be verified with navigation. Handheld retractors are used to ensure visualization down the corridor. Dissection is performed through the external oblique, internal oblique, and transverse abdominal muscles. Then, retroperitoneal fat is identified. Using navigation, we identify the anterior border of the psoas muscle and correct disc level. Once the disc space is reached, it can be cleared of soft tissue with blunt dissection (Fig. 7).

Sequential dilators are placed using triggered EMG 


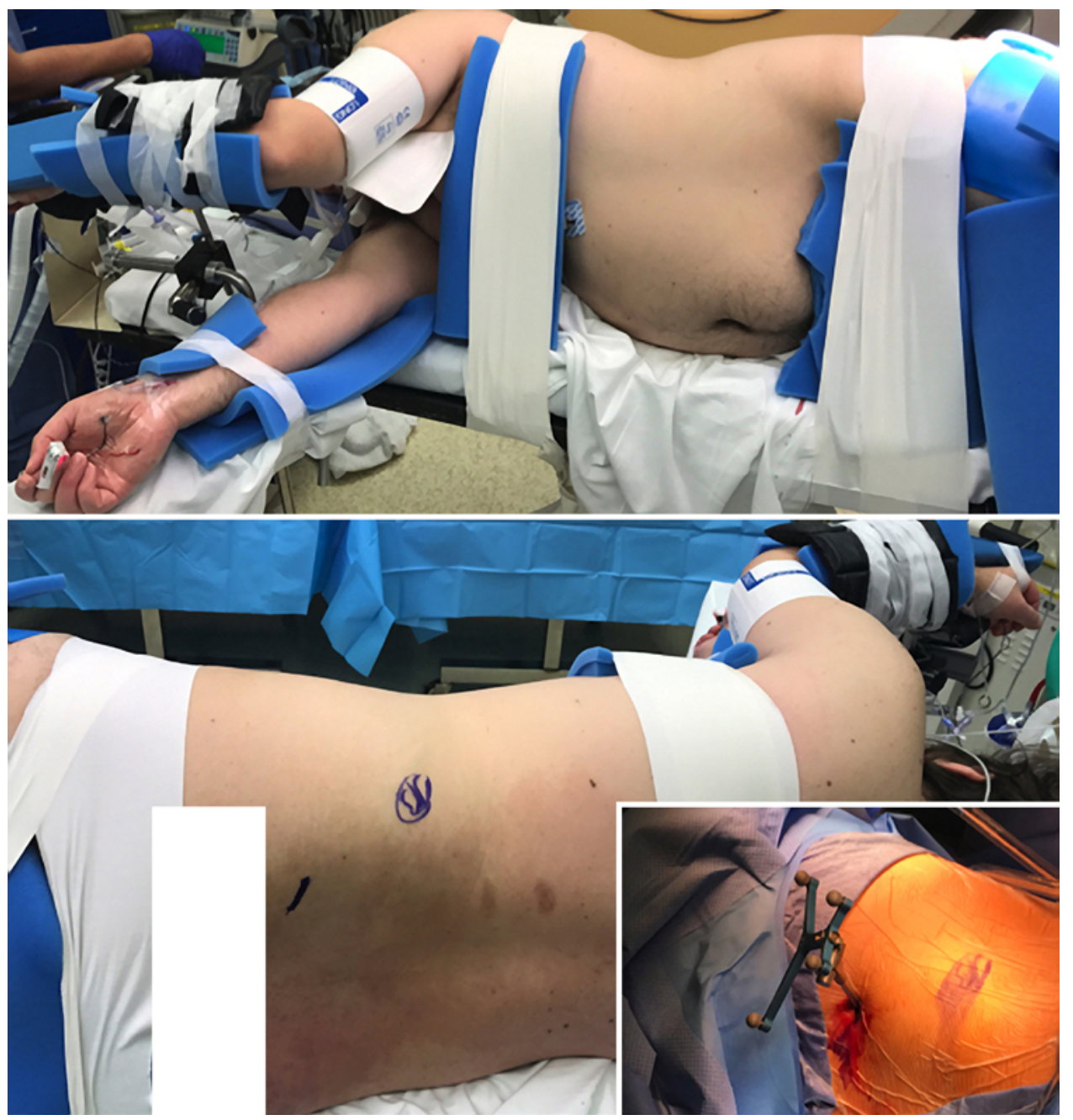

FIG. 4. Positioning for the procedure, with the posterior superior iliac spine (PSIS) marked posteriorly. Inset shows the inserted reference array.
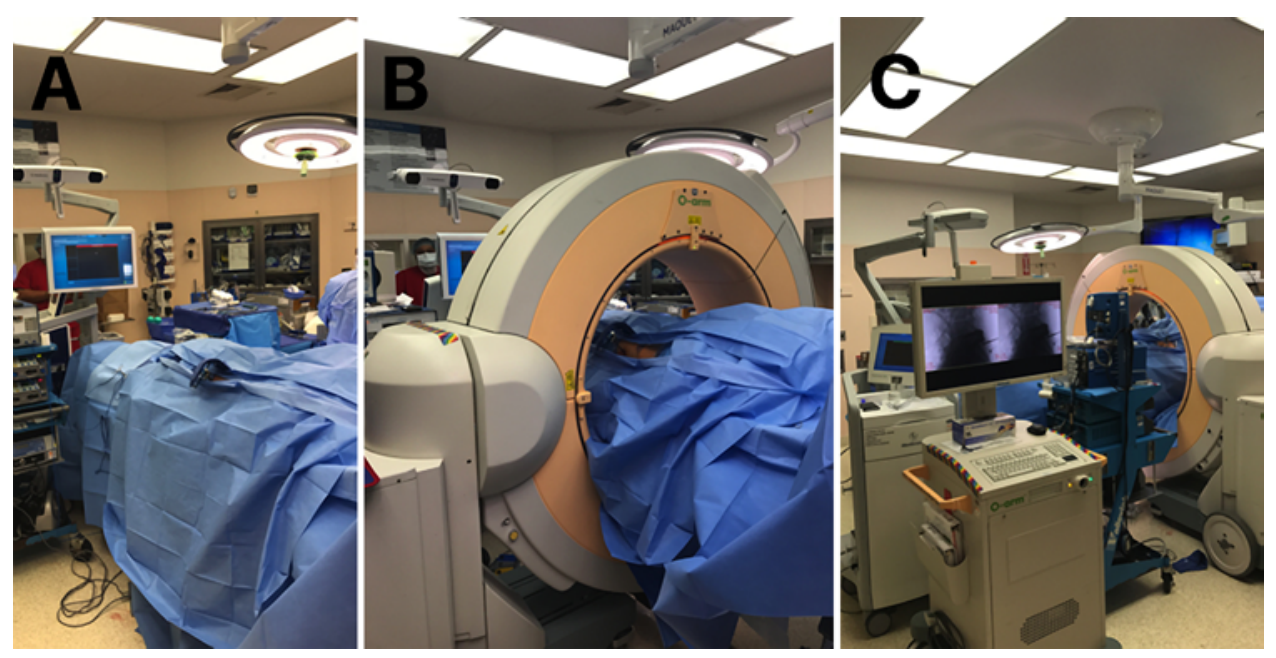

FIG. 5. The room setup as the intraoperative CT is prepared. A: The navigation equipment before the intraoperative CT has moved into place. The navigation computer is at the foot of the bed. The patient has been fully prepared and draped and the navigation array has been placed in the posterior iliac crest. The navigation camera is lifted to have line-of-sight to the array. B: The intraoperative CT in place. C: View from the opposite end of the OR table, showing the control panel for the intraoperative CT. 

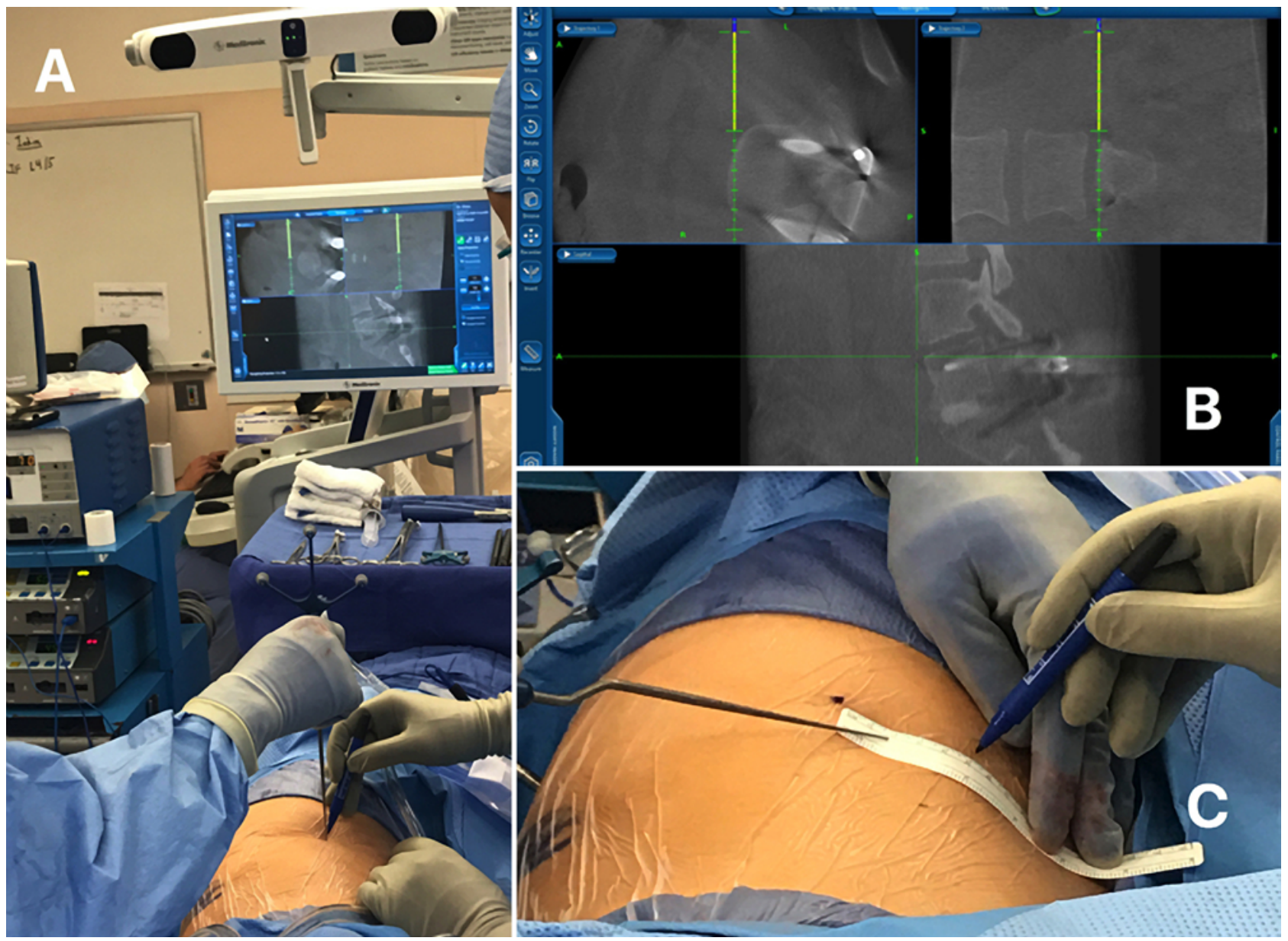

FIG. 6. Incision planning. A: Localization and marking of the pathological disc space from a true lateral position. B: A close-up view of the navigation screen with the virtual extension of the navigation probe localizing the disc space. C: The prepsoas incision being planned $5 \mathrm{~cm}$ from the true lateral mark.

neuromonitoring. Navigation can again be used to confirm the correct disc space and trajectory. The disc annulus is incised with a disc knife. Navigated instruments are used, allowing safe and accurate removal of the disc. When entering the disc space from this angle, a straight oblique trajectory could injure the contralateral nerve root or spinal canal. Thus, it is imperative to obliquely rotate the instruments to a true lateral position before placing them toward the contralateral side. The surgeon must move his or her hand dorsally to correct for this angle. Figure 8 shows the trajectory before and after correcting for the angled approach.

After a thorough discectomy, interbody template trials are used to select the correct cage size. Again, using navigated instruments ensures the correct trajectory and placement. The implant, after being loaded with graft material, is placed with navigational assistance as well (Fig. 9). The ideal location for the implant spans the entire apophyseal ring in the lateral direction.

We routinely check the position of the implant with fluoroscopy to confirm its placement in the event that navigation was not accurate during the procedure.

The wound is then closed in a layered fashion, includ- ing the abdominal fascia, subcutaneous tissue, and skin. We use no. 1 polydioxanone sutures to close the abdominal fascia.

Posterior instrumentation is typically placed unless anterior fixation has already been done. At times, one may choose to stage these surgeries between anterior and posterior portions. This allows for clinical assessment of the indirect foraminal decompression, which can guide the posterior approach. If radicular symptoms have completely resolved, percutaneous fixation only is performed. If the patient has continued radiculopathy, decompression along with instrumentation is performed with the posterior fixation. See Fig. 10 for the postoperative standing radiographs. The procedure is also shown in Video 1.

VIDEO 1. Video clip of navigated prepsoas approach. Copyright

Dean Chou. Published with permission. Click here to view.

\section{Postoperative Management}

The patient is mobilized on the 1st postoperative day. $\mathrm{He}$ or she is limited to a clear liquid diet until flatus. A postoperative ileus is not common but is not unexpected if it occurs. If the posterior instrumentation is staged, pa- 

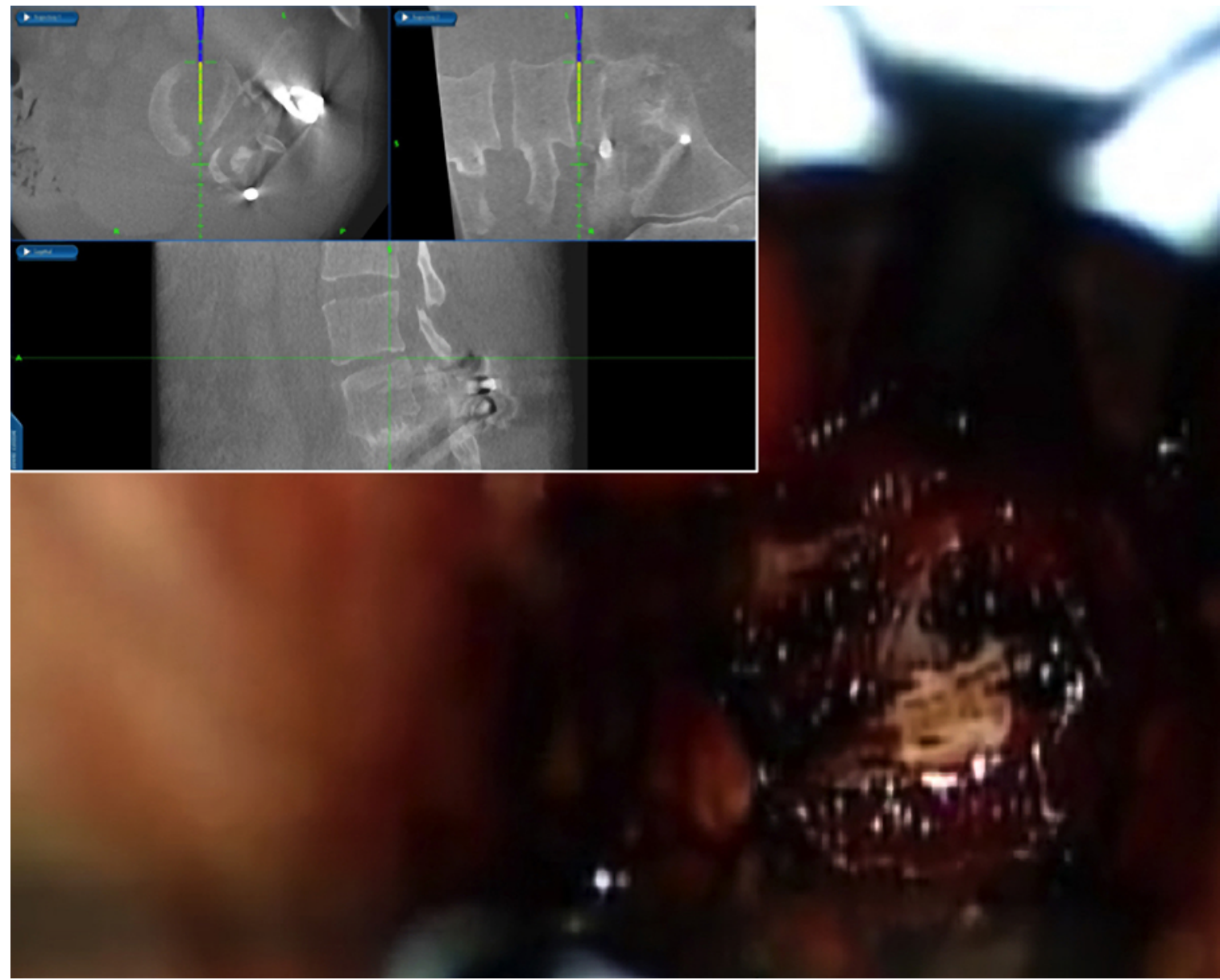

FIG. 7. The retractor has been placed, and the disc space has been bluntly cleared of tissue. Inset shows navigation at this level, confirming correct disc space and trajectory. Navigation shows a projection of the probe (yellow line), and the true navigated position is located at the end of the blue line.

tients can mobilize in the interim, and we encourage this to determine whether there is persistent radicular pain that may need to be addressed via direct dorsal decompression. Patients are discharged home after they are adequately mobilizing, pain has been controlled, and patients are having bowel movements.

\section{Results}

Forty-nine patients underwent a prepsoas approach for lumbar interbody fusion for degenerative disease (D.C. and P.V.M.). Thirty patients were women (61.2\%). The average age was 67.5 years, with a range from 47 to 89 years. The number of fused levels ranged from 1 to 4 , with $51.0 \%$ single-level cases and 6 patients' procedures including the L5-S1 level as well as an interbody fusion from L-2 to L-5. Twenty-eight patients (57.1\%) had undergone prior spinal surgery. Seven patients had stand-alone interbody fusion, 22 had minimally invasively placed posterior instrumentation, and 20 had posterior instrumentation placed via an open approach. Among the 42 patients with posterior instrumentation, the instrumentation was placed on the same day as the interbody fusion in 27 , and the procedures were staged in 15 . See Table 1 for a summary of the patients.

A total of 86 levels were fused in 49 patients. The average follow-up among all patients was 10 months. We included all patients regardless of follow-up since this paper was focused on perioperative morbidity. Many of these patients have not yet reached 1 year of follow-up. We also analyzed a subgroup of 20 patients with at least 1 year of follow-up.

There was 1 surgical complication related to the anterior approach, which was a postoperative psoas hematoma in a 3-level fusion case that resulted in temporary hip flexor weakness. It was treated conservatively, the hematoma resorbed, and the patient's neurological strength returned to normal. There were 6 cases of transient approach-related side effects that were expected for the approach. Three patients had transient left thigh paresthesias that resolved. Three other patients had postoperative ileus that resolved. There were no readmissions within 90 days related to the anterior approach. One patient had persistent radiculopathy, but it was bilateral and believed to be secondary to the adjacent segment above the anterior surgery. The patient in this case underwent posterior decompression at a later date. 

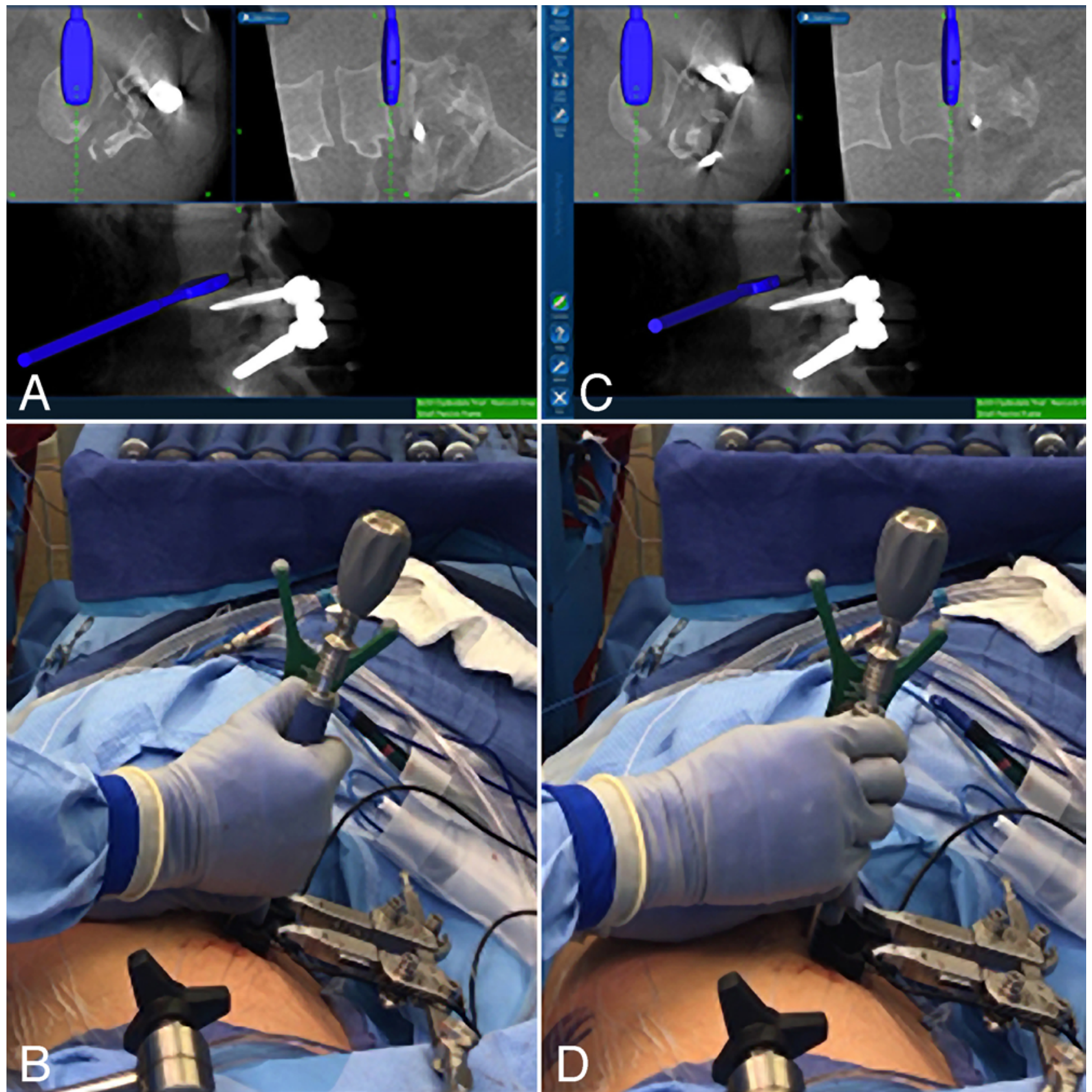

FIG. 8. The discectomy has been completed, and a trial implant is being inserted with navigation screens (A and C) guiding the insertion. The trajectory before the surgeon moves his hand dorsally, correcting for the oblique angle (B). Note that before this correction, the trial trajectory would have taken the implant to the contralateral neural foramen. After correction, the trial has a more optimal trajectory (D).

Only total operating room (OR) time was available for review, so patients who had posterior instrumentation placed during the fusion procedure had significantly higher total OR times than those undergoing staged procedures. For the anterior procedures, the average OR time was 183 minutes. Average blood loss was $258 \mathrm{ml}$. Length of stay was 9.00 days for staged procedures and 4.95 for the nonstaged procedures. The staged procedures averaged 2.1 levels, while the nonstaged procedures averaged 1.6 levels. See Table 2 for perioperative outcomes.
Average back and leg pain scores improved significantly among the group with 1 year of follow-up (Table 3). The VAS back pain scores improved from an average of 7.17 to $4.10(\mathrm{p}=0.001)$ and leg pain scores from 6.15 to 1.20 $(p<0.001)$. There were no hardware failures on follow-up radiographs.

\section{Discussion}

One of the benefits of the prepsoas approach to the lum- 

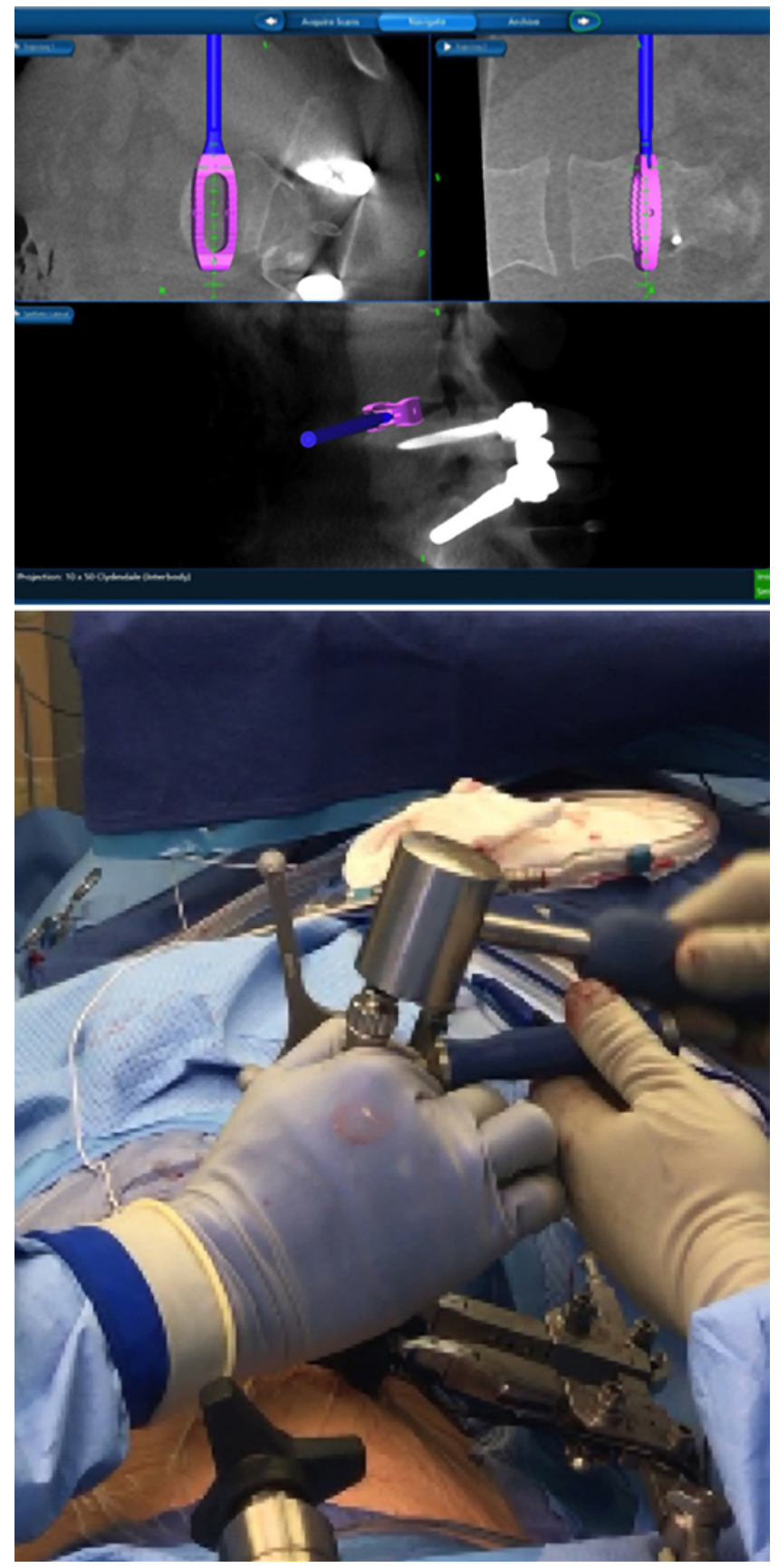

FIG. 9. Insertion of the interbody graft (lower) under navigational guidance (upper). Note that navigation allows for optimal placement of the interbody graft spanning the apophyseal ring.

bar spine is that it obviates the need to dissect through the psoas muscle; not only does this decrease the chance of damage to the psoas muscle itself, but it also may decrease the chance of damage to the nerves that lie within the psoas muscle, such as the femoral nerve. ${ }^{7}$ The transpsoas approach can also expose the cutaneous nerves to the thigh to risk. ${ }^{1}$ Stretching the psoas muscle can lead to hip flexor weakness that is occasionally permanent. A meta-analysis by Joseph et al. showed a $9.4 \%$ rate of temporary and $2.5 \%$ rate of permanent neurological deficits with the transpsoas

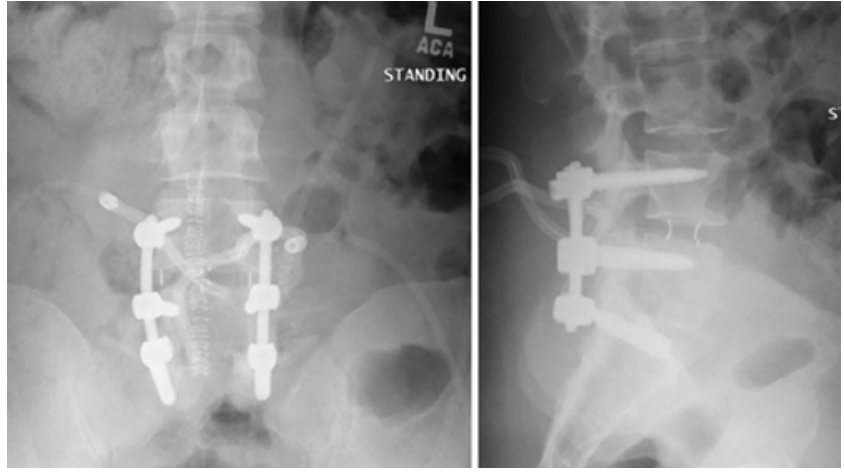

FIG. 10. Postoperative standing radiographs after a staged open procedure for posterior instrumentation.

lateral approach. ${ }^{5}$ In that meta-analysis, there was a $27.1 \%$ rate of sensory deficits. Pseudohernia has been described after the lateral approach.,13 Disruption of the abdominal wall makes this a possibility in the prepsoas approach as well, although we did not encounter it. After opening the fascia of the abdominal wall with electrocautery, we bluntly dissect through the muscles of the abdominal wall. This decreases the risk of transection of the lateral abdominal wall nerves.

Drawbacks to the prepsoas approach include a theoretically higher risk of bowel or vessel injury due to the more anterior corridor. In a review of 179 patients undergoing the prepsoas approach, Silvestre et al. found the most common complications to be incisional pain (4 patients), leg symptoms due to sympathetic chain injury (3), and vascular injury (3). ${ }^{15}$ They had 2 patients with neurological deficit secondary to the procedure, 1 case due to nerve compression from the interbody cage and 1 case presumably from nerve stretching (the patient died 4 months postoperatively from unrelated causes). These authors also had single instances of ileus, peritoneal laceration, cerebrovascular accident, lower extremity ischemia, psoas paresis, groin numbness, and pseudarthrosis.

Another drawback to the prepsoas approach is that the angle of the procedure may be unfamiliar to the surgeon. Typically, when performing a lateral approach, surgeons are coming directly perpendicular to the spinal column, making anatomical landmarks easier to identify to avoid critical structures. In the oblique prepsoas approach, surgeons must keep the angle in mind to avoid injuring the nerves, especially the contralateral nerve root. Navigation can mitigate this risk because the angle and depth of the instruments can be visualized in real time. Another advantage with navigation is the avoidance of radiation exposure to the surgeon. ${ }^{16}$

The technique can only be done from the left side because the vena cava is on the right side. There is no corridor on the right side to perform the prepsoas approach, and the risk of vascular injury precludes a right-sided approach. Thus, in certain cases of scoliosis when the concavity of the L4-5 segment is far below the iliac crest, a left-sided prepsoas approach cannot be used. Either a transpsoas right-sided approach or another type of interbody graft needs to be used in such cases. 
TABLE 1. Preoperative and technique numbers among a series of 49 patients

\begin{tabular}{lc}
\hline \multicolumn{1}{c}{ Parameter } & No. \\
\hline Females & $30(61.2 \%)$ \\
\hline Average age in yrs & 67.5 \\
\hline Patients w/ prior surgery & $28(57.1 \%)$ \\
\hline Levels fused per patient & $25(51 \%)$ \\
\hline 1 & $13(26.5 \%)$ \\
\hline 2 & $9(18.4 \%)$ \\
\hline 3 & $2(4.1 \%)$ \\
\hline 4 & $7(14.3 \%)$ \\
\hline Posterior instrumentation approach & $22(44.9 \%)$ \\
\hline None & $20(40.8 \%)$ \\
\hline Minimally invasive & \\
\hline Open & $27(55.1 \%)$ \\
\hline Posterior instrumentation placed & $15(30.6 \%)$ \\
\hline Same procedure
\end{tabular}

A unique advantage of the prepsoas approach over the direct lateral approach is access to the L5-S1 disc space. It can be done by a trained approach surgeon, and the vascular anatomy must be assessed on preoperative imaging to ensure the presence of an adequate corridor. ${ }^{2}$ Even with an adequate corridor, the rate of vascular injury can be up to $10 \% .^{8}$ The morphology of the sacral slope and iliac crest also needs to be evaluated to ensure that an adequate operative angle can be obtained.

Our patients had good results without any permanent neurological injury. The associated thigh weakness was expected given the approach anterior to the psoas muscle, but it did not appear to be neurogenic in nature in that the weakness resolved to full strength and was not associated with radiculopathy or sensory changes. We did see 1 postoperative hematoma, but this case was performed in a 3-level operation in a patient who had higher than normal oozing intraoperatively. Moreover, cases with an ileus resolved without any sequelae.

Our study is limited in its retrospective design. We wanted to focus on this novel and evolving technique and

TABLE 2. Perioperative outcomes and complications for the prepsoas approach

\begin{tabular}{cc}
\hline \multicolumn{1}{c}{ Parameter } & Value \\
\hline Complication & \\
\hline Psoas hematoma & 1 \\
\hline Thigh sensory changes & 3 \\
\hline Ileus & $306 \mathrm{mins}$ \\
\hline Average total OR time & $258 \mathrm{ml}$ \\
\hline Average EBL & 6.2 days \\
\hline Average length of stay &
\end{tabular}

$\mathrm{EBL}=$ estimated blood loss
TABLE 3. Patient-reported outcomes for 20 patients with at least 1-year follow-up

\begin{tabular}{cccc}
\hline VAS & Preop & Postop & p Value \\
\hline Back pain & 7.17 & 4.10 & 0.001 \\
\hline Leg pain & 6.15 & 1.20 & $<0.001$ \\
\hline
\end{tabular}

its perioperative complications, so we chose to include all of our patients in this study, even though there was short follow-up as this navigated technique was recently developed. However, we also performed a subset analysis of patients with a minimum 1-year follow-up, and among these patients, back and leg pain improved significantly.

\section{Conclusions}

Navigation can aid the surgeon in performing the prepsoas lumbar interbody fusion technique. Navigation is useful for the unfamiliar trajectory associated with this approach and reduces radiation exposure to the surgical team. Given the unfamiliar oblique trajectory, the use of navigation can guide the surgeon in real time in $3 \mathrm{D}$ anatomy and potentially help surgeons who wish to adopt this new approach.

\section{References}

1. Cummock MD, Vanni S, Levi AD, Yu Y, Wang MY: An analysis of postoperative thigh symptoms after minimally invasive transpsoas lumbar interbody fusion. J Neurosurg Spine 15:11-18, 2011

2. Davis TT, Hynes RA, Fung DA, Spann SW, MacMillan M, Kwon B, et al: Retroperitoneal oblique corridor to the L2-S1 intervertebral discs in the lateral position: an anatomic study. J Neurosurg Spine 21:785-793, 2014

3. Galan TV, Mohan V, Klineberg EO, Gupta MC, Roberto RF, Ellwitz JP: Case report: incisional hernia as a complication of extreme lateral interbody fusion. Spine J 12:e1-e6, 2012

4. Gragnaniello C, Seex KA: Anterior to psoas fusion of the lumbar spine. Neurosurg Focus 35 Suppl:Video 13, 2013

5. Joseph JR, Smith BW, La Marca F, Park P: Comparison of complication rates of minimally invasive transforaminal lumbar interbody fusion and lateral lumbar interbody fusion: a systematic review of the literature. Neurosurg Focus 39(4):E4, 2015

6. Mayer HM: A new microsurgical technique for minimally invasive anterior lumbar interbody fusion. Spine (Phila Pa 1976) 22:691-700, 1997

7. Mobbs RJ, Phan K, Malham G, Seex K, Rao PJ: Lumbar interbody fusion: techniques, indications and comparison of interbody fusion options including PLIF, TLIF, MI-TLIF, OLIF/ATP, LLIF and ALIF. J Spine Surg 1:2-18, 2015

8. Molinares DM, Davis TT, Fung DA: Retroperitoneal oblique corridor to the L2-S1 intervertebral discs: an MRI study. J Neurosurg Spine 24:248-255, 2016

9. Moller DJ, Slimack NP, Acosta FL Jr, Koski TR, Fessler RG, Liu JC: Minimally invasive lateral lumbar interbody fusion and transpsoas approach-related morbidity. Neurosurg Focus 31(4):E4, 2011

10. Ohtori S, Orita S, Yamauchi K, Eguchi Y, Ochiai N, Kishida $\mathrm{S}$, et al: Mini-open anterior retroperitoneal lumbar interbody fusion: oblique lateral interbody fusion for lumbar spinal degeneration disease. Yonsei Med J 56:1051-1059, 2015

11. Ozgur BM, Aryan HE, Pimenta L, Taylor WR: Extreme lat- 
eral interbody fusion (XLIF): a novel surgical technique for anterior lumbar interbody fusion. Spine J 6:435-443, 2006

12. Phan K, Maharaj M, Assem Y, Mobbs RJ: Review of early clinical results and complications associated with oblique lumbar interbody fusion (OLIF). J Clin Neurosci 31:23-29, 2016

13. Plata-Bello J, Roldan H, Brage L, Rahy A, Garcia-Marin V: Delayed abdominal pseudohernia in young patient after lateral lumbar interbody fusion procedure: case report. World Neurosurg 91:671.e13-671.e16, 2016

14. Rodgers WB, Gerber EJ, Patterson J: Intraoperative and early postoperative complications in extreme lateral interbody fusion: an analysis of 600 cases. Spine (Phila Pa 1976) 36:26-32, 2011

15. Silvestre C, Mac-Thiong JM, Hilmi R, Roussouly P: Complications and morbidities of mini-open anterior retroperitoneal lumbar interbody fusion: oblique lumbar interbody fusion in 179 patients. Asian Spine J 6:89-97, 2012

16. Villard J, Ryang YM, Demetriades AK, Reinke A, Behr M, Preuss A, et al: Radiation exposure to the surgeon and the patient during posterior lumbar spinal instrumentation: a prospective randomized comparison of navigated versus non-navigated freehand techniques. Spine (Phila Pa 1976) 39:1004-1009, 2014

\section{Disclosures}

Dr. Chou is a consultant for Medtronic and Globus and has received royalties from Globus. Dr. Mummaneni is a consultant for DePuy Spine and Stryker, has ownership in Spinicity/ISD, and has received royalties from DePuy Spine, Thieme Publishing, and Springer Publishing and honoraria from Globus and AOSpine, as well as a grant from AOSpine and ISSG.

\section{Author Contributions}

Conception and design: DiGiorgio, Mummaneni, Chou. Acquisition of data: DiGiorgio, Edwards, Virk, Chou.

Analysis and interpretation of data: DiGiorgio, Edwards, Virk, Mummaneni. Drafting the article: DiGiorgio, Virk. Critically revising the article: Edwards, Virk, Mummaneni, Chou. Reviewed submitted version of manuscript: DiGiorgio, Mummaneni, Chou. Statistical analysis: DiGiorgio. Administrative/technical/material support: Mummaneni, Chou. Study supervision: Mummaneni, Chou.

\section{Supplemental Information}

Videos

Video 1. https://vimeo.com/220807735.

Video Abstract. https://vimeo.com/224938914.

\section{Correspondence}

Anthony M. DiGiorgio, 2020 Gravier St., 7th Fl., New Orleans, LA 70112. email: adigi2@1suhsc.edu. 